\title{
Statine erhöhen Risiko einer Hirnblutung nicht
}

\author{
Ob Statine in der Sekundärprävention des ischämischen Insults zu einem erhöhten Risiko für intra- \\ zerebrale Blutungen führen, wird zurzeit heftig diskutiert. Eine große Analyse gibt Entwarnung.
}

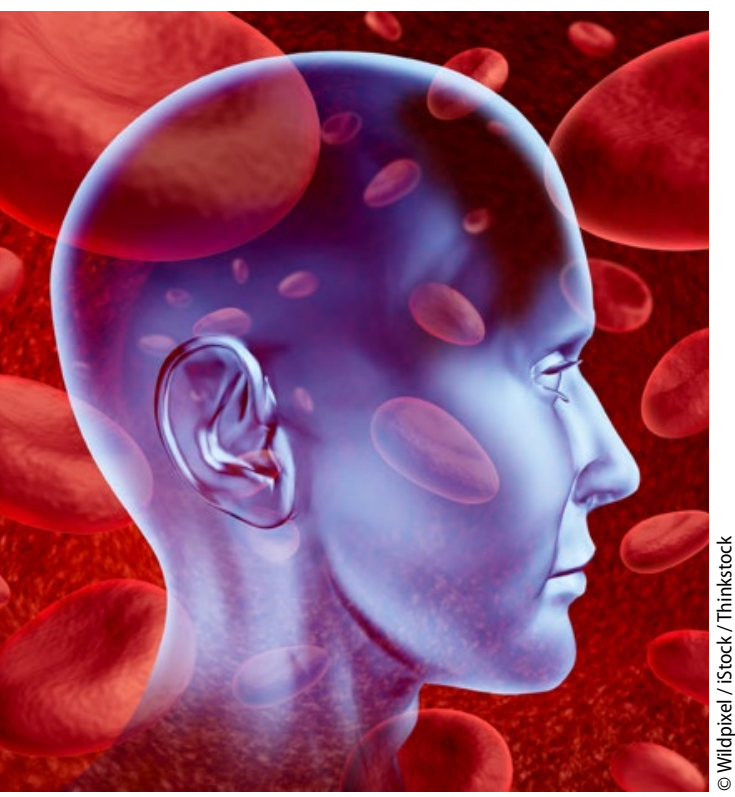

Das Blut soll wieder ungehindert fließen.

_ Hinweise auf eine Neigung zu intrazerebralen Blutungen unter einer Statintherapie hatten sowohl die SPARCL-Studie [Neurology. 2008;70:2364-70] als auch die Heart Protection Study [Lancet.
2004;363:757-67] geliefert. Nun wurden dem Virtual International Stroke Trials Archive (VISTA) Daten von 8.535 Patienten mit ischämischem Insult entnommen, die entweder vor dem Ereignis oder innerhalb von drei Tagen danach eine Statintherapie begonnen hatten. Sie waren im Mittel 70 Jahre alt, 54\% waren Männer. Der mediane Wert auf der NIH-Schlaganfall-Skala lag bei 13. Die Kontrollgruppe bildeten Patienten, die keine Statine erhielten.

Symptomatische intrazerebrale Blutungen traten in der Statingruppe mit einer Odds Ratio (OR) von 1,33 auf, was nicht statistisch signifikant war. Nahm man auch asymptomatische Blutungen hinzu, ergab sich eine ebenfalls nicht signifikante OR von 1,35. Auch der Neubeginn einer Statintherapie nach einem Hirninfarkt erhöhte das Risiko nicht. Eine gesonderte Betrachtung von $\mathrm{Pa}$ tienten, die lysiert wurden, ergab ebenfalls kein erhöhtes Risiko intrazerebraler Blutungen unter Statineinnahme.

Dagegen erschien die Statintherapie in Bezug auf die Mortalität in den 90 Ta- gen nach dem Hirninfarkt von Vorteil. Für Patienten, die zuvor bereits Statine eingenommen hatten, ergab sich ein positiver Trend (Hazard Ratio: 0,84), für den frühen Therapiebeginn nach dem Ereignis sogar ein signifikanter Nutzen (Hazard Ratio: 0,67).

- Scheitz JF et al. Statins and risk of poststroke hemorrhagic complications. Neurology. 2016;86:1590-6

\section{KOMMENTAR}

Die Daten dieser großen Analyse sind sehr beruhigend, da sie zeigen, dass bei Patienten, die einen ischämischen Insult erleiden und zuvor Statine eingenommen haben, kein erhöhtes Risiko intrazerebraler Blutungen besteht. Das gilt insbesondere auch für Patienten, die eine Thrombolyse erhalten.

Für den praktischen Alltag wichtiger ist die Beobachtung, dass auch die im Rahmen der Sekundärprävention früh initiierte Gabe von Statinen das Risiko nicht erhöht. Frühere Studien hatten bereits gezeigt, dass Statine insgesamt die Prognose ischämischer Insulte verbessern - wobei noch unklar ist, ob dies auch für andere Cholesterinsenker gilt.

Prof. Dr. med. H.-C. Diener

Hier steht eine Anzeige.

\author{
Springer
}

